\title{
Magnetic Dissipation in Relativistic Jets
}

\author{
Yosuke Mizuno ${ }^{1, *}$, Jose L. Gómez ${ }^{2}$, Ken-Ichi Nishikawa ${ }^{3}$, Athina Meli ${ }^{4}$, Philip E. Hardee ${ }^{5}$, \\ Luciano Rezzolla ${ }^{1,6}$, Chandra B. Singh ${ }^{7}$ and Elisabete M. de Gouveia Dal Pino ${ }^{7}$ \\ 1 Institute for Theoretical Physics, Goethe University, D-60438 Frankfurt am Main, Germany; \\ rezzolla@itp.uni-frankfurt.de \\ 2 Instituto de Astrofísica de Andalucía (CSIC), 18080 Granada, Spain; jlgomez@iaa.csic.es \\ 3 Department of Physics, University of Alabama in Huntsville, Huntsville, AL 35899, USA; \\ ken-ichi.nishikawa-1@nasa.gov \\ 4 Department of Physics and Astronomy, University of Gent, B-9000 Gent, Belgium; ameli@ulg.ac.be \\ 5 Department of Physics and Astronomy, The University of Alabama, Tuscaloosa, AL 35487, USA; \\ phardee@bama.ua.edu \\ 6 Frankfurt Institute for Advanced Studies, D-60438 Frankfurt am Main, Germany \\ 7 Department of Astronomy (IAG-USP), University of São Paulo, São Paulo SP 05508-090, Brazil; \\ chandratalk@gmail.com (C.B.S.); dalpino@iag.usp.br (E.M.d.G.D.P.) \\ * Correspondence: mizuno@th.physik.uni-frankfurt.de; Tel.: +49-69-7984-7885
}

Academic Editor: Emilio Elizalde

Received: 27 July 2016; Accepted: 26 September 2016; Published: 7 October 2016

\begin{abstract}
The most promising mechanisms for producing and accelerating relativistic jets, and maintaining collimated structure of relativistic jets involve magnetohydrodynamical (MHD) processes. We have investigated the magnetic dissipation mechanism in relativistic jets via relativistic MHD simulations. We found that the relativistic jets involving a helical magnetic field are unstable for the current-driven kink instability, which leads to helically distorted structure in relativistic jets. We identified the regions of high current density in filamentary current sheets, indicative of magnetic reconnection, which are associated to the kink unstable regions and correlated to the converted regions of magnetic to kinetic energies of the jets. We also found that an over-pressured relativistic jet leads to the generation of a series of stationary recollimation shocks and rarefaction structures by the nonlinear interaction of shocks and rarefaction waves. The differences in the recollimation shock structure due to the difference of the magnetic field topologies and strengths may be observable through mm-VLBI observations and space-VLBI mission.
\end{abstract}

Keywords: galaxies: jets; magnetohydrodynamics (MHD); methods: numerical; instabilities; shock waves

\section{Introduction}

Relativistic jets are ubiquitous features of many accreting compact object (mostly black hole) systems. They are associated with X-ray binaries (XRBs) and Gamma-Ray Bursts (GRBs) containing stellar-mass black holes $(\mathrm{BHs})\left(M \sim 1.4-20 M_{\odot}\right)$, and active galactic nuclei (AGNs) with supermassive BHs $\left(M \sim 10^{6}-10^{10} M_{\odot}\right)$.

It is commonly believed that jets are powered and collimated by magnetohydrodynamic (MHD) processes (e.g., [1,2]). General relativistic magnetohydrodynamic (GRMHD) simulations of jet formation (e.g., [3,4]) show development of turbulence via magneto-rotational instability (MRI) [5] and angular momentum transfer in the accretion disk, leading to diffusion of matter and magnetic field inwards, and the generation of unsteady outflows. In general, GRMHD simulations with spinning black holes indicate jet production consisting of a Poynting dominated (in the sense that the energy is transferred predominantly by the electromagnetic field) high Lorentz factor spine jet, and 
a matter dominated, mildly relativistic sheath wind possibly embedded in a sub-relativistic speed disk/coronal wind.

It is thought that relativistic jets on the largest scales are kinetically dominated, i.e., most of the electromagnetic energy is converted to kinetic energy. The means by which magnetic energy is converted to kinetic energy has not been identified, but it is generally thought to involve gradual acceleration by magnetic forces (e.g., [6-8]) and/or non-ideal MHD effects, specifically magnetic reconnection (e.g., [9]). The large scale magnetic field may dissipate if the regular magnetic structure is destroyed as a result of a global MHD instability, the kink instability being the most plausible candidate (e.g., $[10,11])$.

The most promising MHD models for the acceleration and collimation of jets involve the presence of a magnetic field with foot points anchored to a rotating object (an accretion disk or a spinning neutron star or black hole). The dominance of the toroidal component $\left(B_{\phi}\right)$ over the poloidal component $\left(B_{p}\right)$ is a natural consequence in these models. It is well known that current carrying plasma columns containing strong toroidal magnetic fields are unstable to non-axisymmetric perturbations. Among these current-driven (CD) instabilities, the kink mode is the most violent. The kink mode leads to helical displacement of the plasma column and may disrupt the system. Thus, non-linear development of the kink mode could trigger violent magnetic dissipation in relativistic outflows.

On the smallest scales, the rapid observed variability of $\mathrm{X}$-ray/TeV gamma-ray flares with timescales from several minutes to a few hours in blazars pose severe constraints on the particle acceleration timescale and the size of emission region and also provide indirect evidence for instabilities. Here, magnetic reconnection via current driven instability leads to the conversion of Poynting flux to kinetic flux, which provides particle acceleration [12]. Such magnetic dissipation would generate flares from a very small region of a few Schwarzschild radii in size; such flares would be moving very fast, essentially as "min-jets" within the slower jet medium [13,14].

Much of the current discussion lies in whether the $\gamma$-ray flares are produced within the broad-line emission region near the central engine (e.g., [15]), or parsecs away from the central engine (e.g., [16,17]). Results of over three years of simultaneous multi-wavelength observations of 36 blazars show that most $\gamma$-ray flares are simultaneous within errors with the appearance of a new superluminal component or a major outburst in the core of the jet, defined as the bright, compact feature in the upstream end of the jet (e.g., [16,17]). A burst in particle and magnetic energy density is therefore required when jet disturbances cross the radio core in order to produce $\gamma$-ray flares, which can naturally be explained by identifying the radio core with a recollimation shock [18,19].

In this paper, we shortly review our findings for the magnetic dissipation in relativistic jets from recent MHD simulations of CD kink instabilities and recollimation shocks.

\section{Current-Driven Kink Instability}

When jets are magnetically dominated, they are likely to experience $C D$ instability which in turn may drive magnetic reconnection locally. In MHD models for jet collimation and acceleration, a toroidal magnetic field $\left(B_{\phi}\right)$ is wound up due to rotating accretion disk and/or spinning central objects and eventually dominates over the poloidal magnetic field $\left(B_{p}\right)$. The situation with the strong toroidal magnetic field is unstable for CD kink $(m=1)$ mode [10]. The classical Kruskal-Shafranov (KS) instability criterion indicates that the instability develops if the length of a static plasma column is long enough for the field lines to go around the column at least once. One approach to study simple picture of relativistic jet is to consider force-free approximation in which only the charges, currents and fields are considered while the inertia and pressure of the plasma are ignored. This is valid whenever Poynting flux dominated jets are taken into account. Cylindrical force-free jets are kink stable if the axial magnetic field, $B_{z}$, is independent of the radius of plasma column [20] but are kink unstable if $B_{z}$ decreases with increasing distance from the axis $[10,21]$. 
The temporal development of the CD kink instability in relativistic cylindrical jets using periodic computational box has been explored. The instability of a helically magnetized relativistic non-rotating force-free static plasma column has been studied and has shown that the initial configuration is strongly distorted but not disrupted by the CD kink instability [22]. The growth rate and nonlinear evolution of the $C D$ kink instability depends moderately on the radial density profile and strongly on the radial magnetic pitch profile. This work was further extended to investigate the influence of a velocity shear surface on the development of the CD kink instability in a sub-Alfvénic non-rotating jet [23]. It was found that the helically distorted density structure propagated along the jet with the flow speed and the structure dependent on the ratio between the radius of the velocity shear surface and the characteristic radius of the helically twisted force-free magnetic field (the radius of maximum toroidal magnetic field). The influence of the jet rotation and the differential motion on the CD kink instability was studied for rotating helically magnetized relativistic jet with radially decreasing density profile and it was found that the development of the instability depends on the lateral distribution of the poloidal magnetic field [24]. If the poloidal field significantly decreases outward from the axis, then the initial small perturbations grow strongly, then nonlinear interaction with multiple axial modes of instability eventually disrupts the initial cylindrical configuration. When the profile of the poloidal field is shallow, the instability develops slowly and eventually saturates.

Recently, we have investigated the influence of the radial density profile on the spatial development of the CD kink instability along helically magnetized rotating, relativistic cylindrical jets using a non-periodic computational box and a precessional perturbation at the inlet triggering the growth of the kink instability [25]. The upper panels of Figure 1 shows the 3D density structure of a CD kink instability in magnetized rotating, relativistic cylindrical jets with decreasing and increasing radial density profiles. It shows that the helical kink develops and grows continuously near the jet inlet, and also propagates downstream from the jet, exciting the CD kink instability in both cases. The kink structure propagates downstream nearly with the flow speed. The growth rate of the kink instability itself does not depend on the angular velocity amplitude $\Omega_{0}$ as suggested by periodic box simulations of temporal kink growth [24], but depends on the radial density profile. The decreasing density cases exhibit faster growth of the kink instability, as reported in spatial growth studies of non-rotating cases [26]. The lower panels of Figure 1 depicts arrows that identify the locations where the current density $\mathbf{J} \propto \nabla \times \mathbf{B}$ attains the largest intensities. They trace the locations of inversion of the polarity of the magnetic field lines and therefore of potential sites for magnetic reconnection. We clearly see that the location of the maximum kink amplitude matches the maximum value of $c u r l B$ in both cases. Hence, these regions are the possible sites for magnetic energy dissipation through magnetic reconnection, which can contribute to the increase in kinetic energy of the jet flow and also allow for efficient particle acceleration (e.g., [27]). Takamoto et al. [28] have investigated fast magnetic reconnection induced by turbulence in the relativistic regime considering both incompressible and compressible flows numerically and found that the reconnection rate is independent of the plasma resistivity as in the non-relativistic case [29]. They measured reconnection rate values depending on the magnetization parameter $\sigma$ of the flow. We also make a quite rough estimate of the reconnection rate in the magnetic reconnection sites identified from the simulations. Considering the values of the components of $\mathbf{B}$ and $\mathbf{v}$ in these regions, we obtained similar reconnection rates to the values obtained by Takamoto et al. [28]. This further indicates that the turbulence due to the CD kink instability in the relativistic jets is able to induce fast magnetic reconnection locally and thus may provide potential sites for particle acceleration by reconnection and non-thermal high energy radiation [30]. 
Decreasing density $\Omega_{0}=2, \mathrm{t}=100$
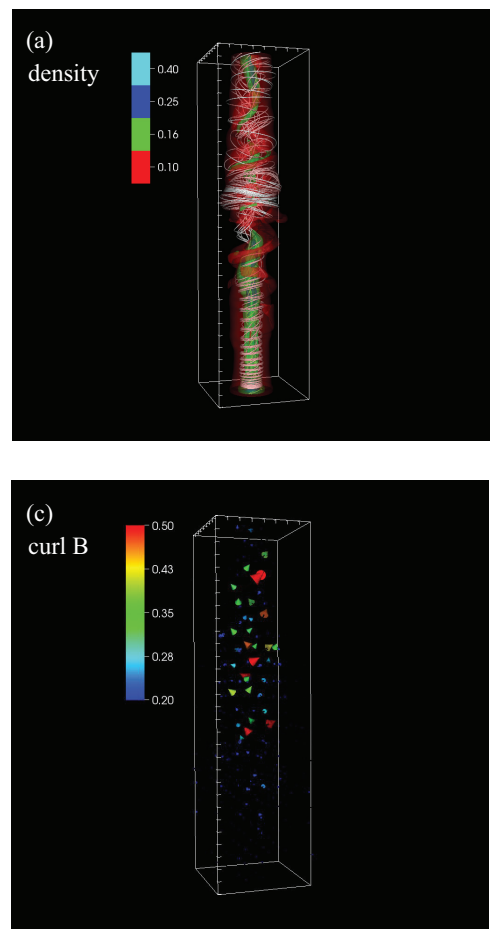

Increasing density $\quad \Omega_{0}=2, \mathrm{t}=150$
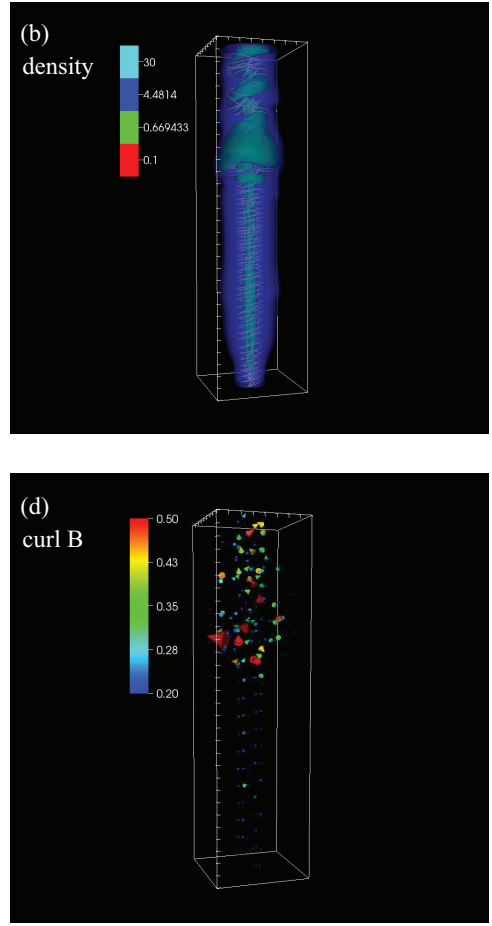

Figure 1. upper: 3D density isosurfaces and lower: the locations of maximum current density, curlB for $(\mathbf{a}, \mathbf{c})$ the decreasing density model with $\Omega_{0}=1$ at $t_{s}=100$ and $(\mathbf{b}, \mathbf{d})$ the increasing density model with $\Omega_{0}=2$ at $t_{s}=150$ of CD kink instability in relativistic rotating jets. The solid lines correspond to the magnetic field lines. The current density traces the regions where magnetic reconnection may occur, at the same time. Figure taken from [25].

\section{Recollimation Shock}

Most of the observed quasi-stationary features appear in the innermost jet regions by Very Long Baseline Interferometry (VLBI) observations of AGN jets suggesting that they could be associated with recollimation shocks produced by a pressure mismatch between the jet and the external medium (e.g., [31]). Recollimation shocks have also been found at hundreds of parsecs from the central objects in several AGN jets (e.g., [32]).

When a jet propagates through an ambient medium, the pressure mismatch between the jet and the ambient medium naturally arises as a result of the ambient pressure decrease. The pressure mismatch drives a radial oscillating motion of the jet and multiple recollimation regions inside the jet (e.g., [19]). If a significant rarefaction wave is produced by the recollimation and propagates into the jet interior, the plasma thermal energy can be converted into jet kinetic energy, increasing considerably the jet Lorentz factor. This is a purely relativistic effect, also referred to as the Aloy-Rezzolla (AR) booster [33], which takes place in relativistic flows with a large tangential velocity discontinuity. This boosting mechanism is very basic and has been confirmed by a number of studies in hydrodynamical and magnetized jets (e.g., [34]).

We have performed 2D RMHD simulations of the propagation of a non-equilibrium over-pressured relativistic jets leading to the generation of a series of recollimation shocks and rarefaction waves. Figure 2 shows 2D plots of the rest-mass density, of the gas pressure, and of the Lorentz factor for the helical magnetic field case. Similar to the other magnetic field topologies (purely axial and toroidal fields, not shown here), in this case the over-pressured jet produces an initially weak conical shock that propagates into the ambient medium and a conical rarefaction wave that propagates into the jet. Through the AR booster mechanism, a conversion of thermal energy to jet kinetic energy takes place across the rarefaction wave, with a consequent acceleration of the flow 
from initial jet Lorentz factor $\gamma_{j, 0}=3$ to $\gamma_{j} \simeq 5$. Although the pitch considered here is less than one, so that the toroidal magnetic field is larger than the axial one at least initially, the effective behaviour of the plasma is closer to the one seen in the case of an axial magnetic field than in the case of a toroidal magnetic field. The presence of a helical magnetic field in the jet leads to a rather complex behaviour in both the recollimation shock and rarefaction structure. This global behaviour is clearly influenced in part by our choice for the initial pitch.

\section{helical $B, t=200$}
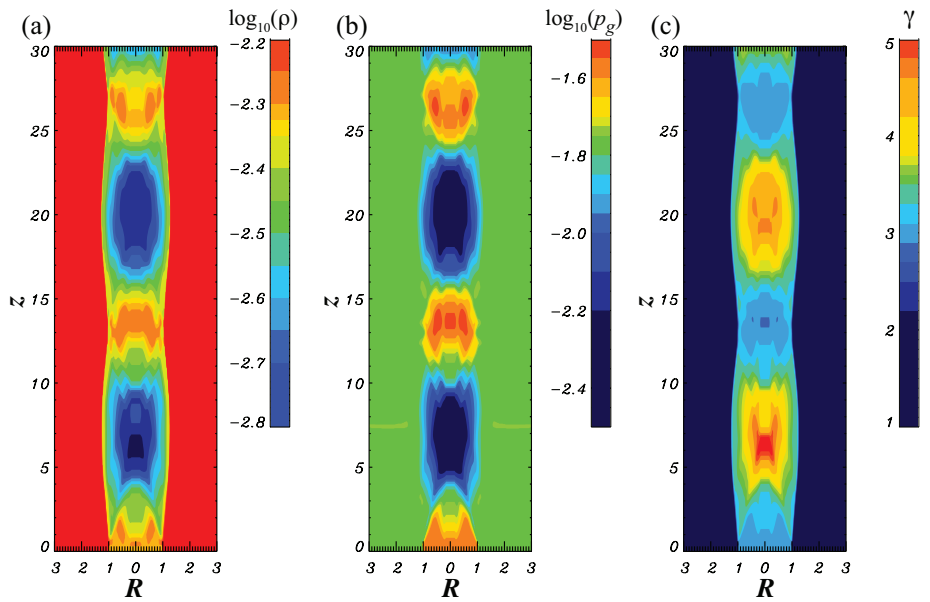

Figure 2. 2D plots of: (a) the rest-mass density; (b) the gas pressure; and (c) the Lorentz factor for 2D RMHD simulations of recollimation shock in over-pressured relativistic jet with helical magnetic field. Figure taken from [35].
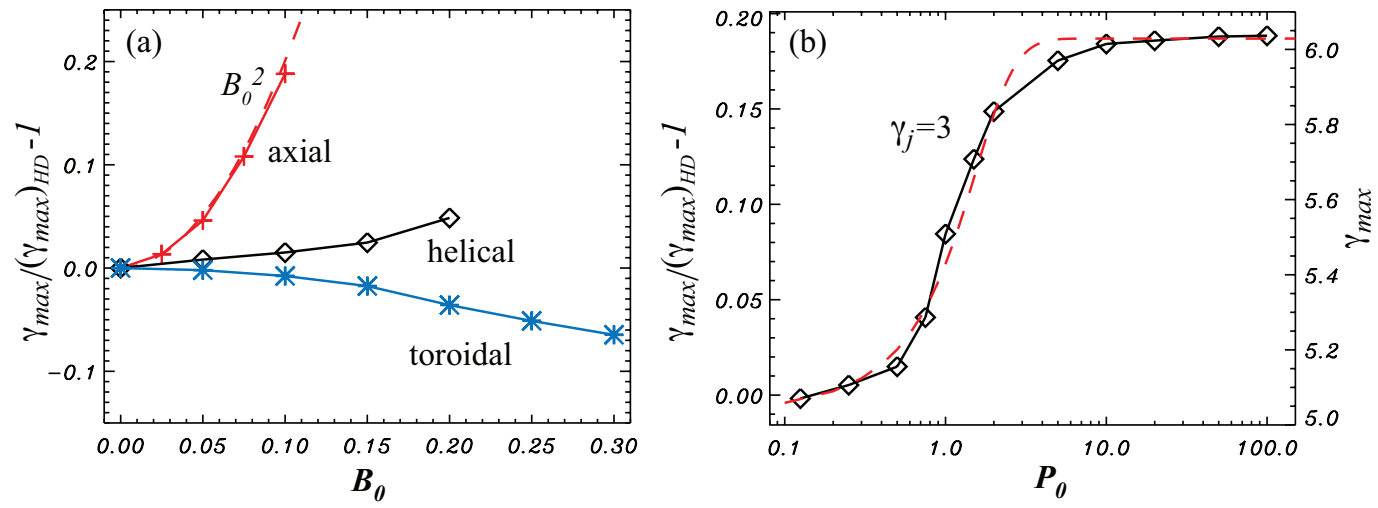

Figure 3. Left panel: Dependence of the relative difference of the maximum Lorentz factor relative to the purely hydrodynamic case $\gamma_{\max } /\left(\gamma_{\max }\right)_{\mathrm{HD}}-1$. All quantities are shown as a function of the initial magnetic field strength $B_{0}$ in the case of axial (red crosses), toroidal (blue stars), and helical magnetic fields (black diamonds) with initial magnetic pitch $P_{0}=0.5$. The red dashed line indicates a quadratic fit. Right panel: Dependence of the maximum Lorentz factor $\gamma_{\max }$ (right vertical axis) and relative difference of the maximum Lorentz factor relative to the purely hydrodynamic case $\gamma_{\max } /\left(\gamma_{\max }\right)_{\mathrm{HD}}-1$ (left vertical axis) as a function of the initial magnetic pitch $P_{0}$. The initial jet Lorentz is set to $\gamma_{j}=3$. The red dashed line indicates the fitting with a hyperbolic tangent function. Figure taken from [35].

The left panel of Figure 3 reports the dependence of the relative increase of the Lorentz factor with respect to the purely hydrodynamical evolution, namely, $\Delta \gamma_{\max } /\left(\gamma_{\max }\right)_{\mathrm{HD}} \equiv \gamma_{\max } /\left(\gamma_{\max }\right)_{\mathrm{HD}}-1$, as a function of the initial magnetic field. Obviously, this quantity can either be positive or negative and provides a direct measure of the fractional boost. Shown with different symbols are the different 
magnetic-field topologies with crosses referring to the axial magnetic field, diamonds to the helical magnetic-field, and star crosses to the toroidal magnetic-field. From the results, axial and helical initial magnetic fields lead to Lorentz boosts that are larger than in the hydrodynamical case, while the opposite is true for purely toroidal magnetic fields, for which an acceleration is still present but this is smaller than in the hydrodynamical case. The origin of this different behaviour has to be found in the fact that an axial magnetic field adds an effective gas pressure and results in larger rest-mass density and pressure gradients across the rarefaction waves induced downstream of the inlet. In turn, the AR booster translates these stronger waves into larger accelerations of the flow. It is also instructive that, in the case of a purely axial magnetic field, the behaviour of the relative boost has a simple quadratic dependence on the initial magnetic field. This is simply because the relative boost scales as $\Delta \gamma_{\max } /\left(\gamma_{\max }\right)_{\mathrm{HD}} \propto p / p_{g}-1=p_{m} / p_{g} \propto B_{z}^{2}$. This is confirmed by the very good match between the numerical data and a quadratic fit, which is indicated with a red dashed line.

In the case of a helical magnetic field, we have an additional degree of freedom represented by the initial magnetic pitch, $P_{0}=R B_{z} / R_{j} B_{\phi}$. By suitably choosing the initial pitch, it is possible to scan the range of possible magnetic-field configurations, which range from an essentially toroidal magnetic field for $P_{0} \ll 1$ to an axial axial magnetic field for $P_{0} \gg 1$. The right panel of Figure 3 shows $\Delta \gamma_{\max } /\left(\gamma_{\max }\right)_{\mathrm{HD}}$ as a function of the initial pitch $P_{0}$ with fixed initial magnetic field strength $B_{0}=0.1$. Interestingly, we found that the relative increase in the maximum Lorentz factor has a very clear dependence with the pitch, smoothly joining the two extreme cases of a toroidal and axial magnetic fields, respectively. Furthermore, the transition between the two regimes takes place at $P_{0} \geq 1$ saturating to the axial field case when $P_{0} \simeq 10$. Finally, the dependence can be accurately approximated with a simple expression of the type $\gamma_{\max } \simeq c_{1}+c_{2} \tanh \left[c_{3}\left(P_{0}-1.0\right)\right]$, where $c_{1} \simeq 5.43, c_{2} \simeq 0.6$, and $c_{3} \simeq 0.8$, and is indicated with the red dashed line.

\section{Summary and Conclusions}

In this paper, we have shortly reviewed our recent work related on the magnetic dissipation in relativistic jets. Through numerical studies of CD kink instability in relativistic jets, the growth of CD kink instability becomes the potential trigger mechanism of the fast magnetic reconnection in relativistic jets through development of turbulence locally and can govern the transformation of magnetic to kinetic energy, thus providing an efficient way to power and accelerate particles in relativistic jets. We note here that it is important that relativistic jets are not disrupted entirely by the CD instability and local magnetic dissipation continues to accelerate particles.

Multiple stationary recollimation shock and rarefaction structures are produced along the jet by the nonlinear interaction of shocks and rarefaction waves in the propagation of an over-pressured magnetized relativistic jet. The propagation of rarefaction waves in the jet leads to the conversion of the plasma thermal energy into jet kinetic energy with significant boost of the jet Lorentz factor [33]. The presence of magnetic field modifies the recollimation shock strength and structure which may affect in a significant way the signatures of stationary components seen in several AGN jets, especially in polarized flux. Studying in detail the structure in these stationary components for a direct comparison with our simulations requires however, resolving the jet structure across the jet width. This can be achieved through mm-VLBI observations by GMVA (Global Millimeter VLBI Array) [36], the Event Horizon Telescope and the Black Hole Cam project [37,38], and the space VLBI mission RadioAstron [39].

Acknowledgments: Support comes the ERC Synergy Grant “BlackHoleCam - Imaging the Event Horizon of Black Holes" (Grant 610058). J. L. G. acknowledges support from the Spanish Ministry of Economy and Competitiveness grant AYA2013-40825-P. K. N. and P. H. acknowledge support by NSF awards AST-0908010 and AST-0908040, and by NASA awards NNX09AD16G, NNX12AH06G, NNX13A P-21G, and NNX13AP14G. A. M. acknowledges support from the Scientific Research (FWO) and the Belgian Federal Science Policy Office (Belspo). C.B.S. acknowledges support from the Brazilian agency FAPESP (2013/09065-8). E.M.G.D.P. ackowledges support from FAPESP (2013/10559-5) and CNPq (306598/2009-4) grants. The simulations were performed on Pleiades at NASA, on SR16000 at Kyoto University, on LOEWE at the Goethe University Frankfurt, and on LAi - Laboratory of Astroinformatics at IAG/USP and NAT/Unicsul, (FAPESP grant 2009/54006-4). 
Author Contributions: Y. Mizuno: Performed simulations, analyzed the data and prepared the manuscript; J. L.Gómez: Contributed on the simulation setup and comparisons with observations; K.-I. Nishikawa: Contribution for discussions on this research; A. Meli: Critical reading and discussions on this research; P. E. Hardee: Theoretical contributions for this research; L. Rezzolla: Critical contributions for physical interpretation of simulation results; C. B. Singh: Performed some of simulations and analyzed the data for this research; E. M. de Gouveia Dal Pino: Contributions for physical interpretation for this research.

Conflicts of Interest: The authors declare no conflict of interest.

\section{References}

1. Blandford, R.D.; Payne, D.G. Hydromagnetic flows from accretion discs and the production of radio jets. Mon. Not. R. Astron. Soc. 1982, 199, 883-903.

2. Blandford, R.D.; Znajek, R.L. Electromagnetic extraction of energy from Kerr black holes. Mon. Not. R. Astron. Soc. 1977, 179, 433-456.

3. McKinney, J.C. General relativistic magnetohydrodynamic simulations of the jet formation and large-scale propagation from black hole accretion systems. Mon. Not. R. Astron. Soc. 2006, 368, 1561-1582.

4. McKinney, J.C.; Blandford, R.D. Stability of relativistic jets from rotating, accreting black holes via fully three-dimensional magnetohydrodynamic simulations. Mon. Not. R. Astron. Soc. 2009, 39, L126-L130.

5. Balbus, S.A.; Hawley, J.F. Instability, turbulence, and enhanced transport in accretion disks. Rev. Mod. Phys. 1998, 70, 1-53.

6. Beskin, V.S.; Nokhrina, E.E. The effective acceleration of plasma outflow in the paraboloidal magnetic field. Mon. Not. R. Astron. Soc. 2006, 367, 375-386.

7. Lyubarsky, Y. Asymptotic Structure of Poynting-Dominated Jets. Astrophys. J. 2009, 698, 1570-1589.

8. Lyubarsky, Y.E. Transformation of the Poynting flux into kinetic energy in relativistic jets. Mon. Not. R. Astron. Soc. 2010, 402, 353-361.

9. McKinney, J.C.; Uzdensky, D.A. A reconnection switch to trigger gamma-ray burst jet dissipation. Mon. Not. R. Astron. Soc. 2012, 419, 573-607.

10. Begelman, M.C. Instability of Toroidal Magnetic Field in Jets and Plerions. Astrophys. J. 1998, 493, $291-300$.

11. Giannios, D.; Spruit, H.C. The role of kink instability in Poynting-flux dominated jets. Astron. Astrophys. 2006, 450, 887-898.

12. Sikora, M.; Begelman, M.C.; Madejski, G.M.; Lasota, J.-P. Are Quasar Jets Dominated by Poynting Flux? Astrophys. J. 2005, 625, 72-77.

13. Begelman, M.C.; Fabian, A.C.; Rees, M.J. Implications of very rapid TeV variability in blazars. Mon. Not. R. Astron. Soc. 2008, 384, L19-L23.

14. Giannios, D.; Uzdensky, D.A.; Begelman, M.C. Fast TeV variability in blazars: Jets in a jet. Mon. Not. R. Astron. Soc. 2009, 395, L29-L33.

15. Tavecchio, F.; Ghisellini, G.; Bonnoli, G.; Ghirlanda, G. Constraining the location of the emitting region in Fermi blazars through rapid $\gamma$-ray variability. Mon. Not. R. Astron. Soc. 2010, 405, L94-L98.

16. Marscher, A.P.; Jorstad, S.G.; Larionov, V.M.; Aller, M.F.; Aller, H.D.; Lähteenmäki, A.; Agudo, I.; Smith, P.S.; Gurwell, M.; Hagen-Thorn, V.A.; et al. Probing the Inner Jet of the Quasar PKS 1510-089 with Multi-Waveband Monitoring During Strong Gamma-Ray Activity. Astrophys. J. Lett. 2010, 710, L126-L131.

17. Agudo, I.; Jorstad, S.G.; Marscher, A.P.; Larionov, V.M.; Gómez, J.L.; Lähteenmäki, A.; Gurwell, M.; Smith, P.S.; Wiesemeyer, H.; Thum, C.; et al. Location of $\gamma$-ray Flare Emission in the Jet of the BL Lacertae Object OJ287 More than 14 pc from the Central Engine. Astrophys. J. Lett. 2011, 726, L13.

18. Gómez, J.L.; Martí, J.M.A.; Marscher, A.P.; Ibáñez, J.M.A.; Marcaide, J.M. Parsec-Scale Synchrotron Emission from Hydrodynamic Relativistic Jets in Active Galactic Nuclei. Astrophys. J. Lett. 1995, 449, L19-L21.

19. Gómez, J.L.; Martí, J.M.; Marscher, A.P.; Ibáñez, J.M.; Alberdi, A. Hydrodynamical Models of Superluminal Sources. Astrophys. J. Lett. 1997, 482, L33-L36.

20. Istomin, Y.N.; Pariev, V.I. Stability of a relativistic rotating electron-positron jet: Non-axisymmetric perturbations. Mon. Not. R. Astron. Soc. 1996, 281, 1-26.

21. Lyubarskii, Y.E. Kink instability of relativistic force-free jets. Mon. Not. R. Astron. Soc. 1999, 308, $1006-1010$.

22. Mizuno, Y.; Lyubarsky, Y.; Nishikawa, K.-I.; Hardee, P.E. Three-Dimensional Relativistic Magnetohydrodynamic Simulations of Current-Driven Instability. I. Instability of a Static Column. Astrophys. J. 2009, 700, 684-693. 
23. Mizuno, Y.; Hardee, P.E.; Nishikawa, K.-I. Three-dimensional Relativistic Magnetohydrodynamic Simulations of Current-driven Instability with a Sub-Alfvénic Jet: Temporal Properties. Astrophys. J. 2011, 734, 19.

24. Mizuno, Y.; Lyubarsky, Y.; Nishikawa, K.-I.; Hardee, P.E. Three-dimensional Relativistic Magnetohydrodynamic Simulations of Current-driven Instability. III. Rotating Relativistic Jets. Astrophys. J. 2012, 757, 16.

25. Singh, C.B.; Mizuno, Y.; de Gouveia Dal Pino, E.M. Spatial Growth of Current-driven Instability in Relativistic Rotating Jets and the Search for Magnetic Reconnection. Astrophys. J. 2016, 824, 48.

26. Mizuno, Y.; Hardee, P.E.; Nishikawa, K.-I. Spatial Growth of the Current-driven Instability in Relativistic Jets. Astrophys. J. 2014, 784, 167.

27. Kowal, G.; de Gouveia Dal Pino, E.M.; Lazarian, A. Particle Acceleration in Turbulence and Weakly Stochastic Reconnection. Phys. Rev. Lett. 2012, 108, 241102.

28. Takamoto, M.; Inoue, T.; Lazarian, A. Turbulent Reconnection in Relativistic Plasmas and Effects of Compressibility. Astrophys. J. 2015, 815, 16.

29. Kowal, G.; Lazarian, A.; Vishniac, E.T.; Otmianowska-Mazur, K. Numerical Tests of Fast Reconnection in Weakly Stochastic Magnetic Fields. Astrophys. J. 2009, 700, 63-85.

30. De Gouveia Dal Pino, E.M.; Kowal, G. Particle Acceleration by Magnetic Reconnection. In Magnetic Fields in Diffuse Media; Lazarian, A., de Gouveia Dal Pino, E.M., Melioli, C., Eds.; Astrophysics and Space Science Library; Springer: Berlin/Heidelberg, Germany, 2015; pp. 373-396.

31. Cohen, M.H.; Meier, D.L.; Arshakian, T.G.; Homan, D.C.; Hovatta, T.; Kovalev, Y.Y.; Lister, M.L.; Pushkarev, A.B.; Richards, J.L.; Savolainen, T. Studies of the Jet in Bl Lacertae. I. Recollimation Shock and Moving Emission Features. Astrophys. J. 2014, 787, 151.

32. Asada, K.; Nakamura, M. The Structure of the M87 Jet: A Transition from Parabolic to Conical Streamlines. Astrophys. J. Lett. 2012, 745, L28.

33. Aloy, M.A.; Rezzolla, L. A Powerful Hydrodynamic Booster for Relativistic Jets. Astrophys. J. Lett. 2006, 640, L115-L118.

34. Mizuno, Y.; Hardee, P.; Hartmann, D.H.; Nishikawa, K.-I.; Zhang, B. A Magnetohydrodynamic Boost for Relativistic Jets. Astrophys. J. 2008, 672, 72-82.

35. Mizuno, Y.; Gómez, J.L.; Nishikawa, K.-I.; Meli, A.; Hardee, P.E.; Rezzolla, L. Recollimation Shocks in Magnetized Relativistic Jets. Astrophys. J. 2015, 809, 38.

36. Krichbaum, T.P.; Roy, A.; Wagner, J.; Rottmann, H.; Hodgson, J.A.; Bertarini, A.; Alef, W.; Zensus, J.A.; Marscher, A.; Jorstad, S.G.; et al. Zooming towards the Event Horizon - mm-VLBI Today and Tomorrow. In Proceedings of the 11th EVN Symposium 055. Available online: http://pos.sissa.it/archive/conferences/178/055/11th\%20EVN\%20Symposium_055.pdf (accessed on 20 September 2016)

37. Fish, V.L.; Johnson, M.D.; Lu, R.-S.; Doeleman, S.S.; Bouman, K.L.; Zoran, D.; Freeman, W.T.; Psaltis, D.; Narayan, R.; Pankratius, V.; et al. Imaging an Event Horizon: Mitigation of Scattering toward Sagittarius A*. Astrophys. J. 2014, 795, 134.

38. Goddi, C.; Falcke, H.; Kramer, M.; Rezzolla, L.; Brinkerink, C.; Bronzwaer, T.; Deane, R.; De Laurentis, M.; Desvignes, G.; Davelaar, J.R.J.; et al. BlackHoleCam: Fundamental physics of the Galactic center. Int. J. Mod. Phys. D 2016, submitted.

39. Gomez, J.L.; Lobanov, A.P.; Bruni, G.; Kovalev, Y.Y.; Marscher, A.P.; Jorstad, S.G.; Mizuno, Y.; Bach, U.; Sokolovsky, K.V.; Anderson, J.M.; et al. Probing the Innermost Regions of AGN Jets and Their Magnetic Fields with RadioAstron. I. Imaging BL Lacertae at 21 Microarcsecond Resolution. Astrophys. J. 2016, 817, 96.

(C) 2016 by the authors; licensee MDPI, Basel, Switzerland. This article is an open access article distributed under the terms and conditions of the Creative Commons Attribution (CC-BY) license (http://creativecommons.org/licenses/by/4.0/). 